\title{
Metodología basada en el Modelo de Referencia para Cadenas de Suministro para Analizar el Proceso de producción de Biodiesel a partir de Higuerilla
}

\author{
Fernando Salazar ${ }^{(1)}$, Judith Cavazos ${ }^{(2)}$, José L. Martínez ${ }^{(2)}$ \\ (1) Departamento de Ingeniería Industrial, Pontificia Universidad Javeriana, Bogotá D.C.- \\ Colombia. \\ (2) Centro Interdisciplinario de Posgrado, Investigación y Consultoría, Universidad Popular \\ Autónoma del Estado de Puebla -México (e-mail: fsalazar.upaep@gmail.com)
}

Recibido Jul. 05, 2011; Aceptado Sep. 02, 2011; Versión Final recibida Nov. 15, 2011

\begin{abstract}
Resumen
Se presenta un análisis para la producción de Biodiesel a partir de Higuerilla usando el Modelo de Referencia para Cadenas de Suministro (SCOR). Se identificaron los niveles del proceso, indicadores clave de desempeño, atributos y operaciones logísticas que se llevan a cabo en toda la cadena de la producción de biodiesel de higuerilla. La utilidad de la aplicación del modelo SCOR se determina por la identificación que logra de los componentes de la cadena, evidenciando fortalezas y debilidades que presenta en las relaciones externas e internas de la logística. Desde esta perspectiva se observó que la aplicación del modelo SCOR hace más eficiente las operaciones logísticas a lo largo de la cadena.
\end{abstract}

Palabras Clave: SCOR, biodiesel, biocombustibles, higuerilla, cadenas de suministro

\section{Methodology based on the Supply Chain Operations Reference Model to Analyze the Production Process of Biodiesel from Castor Oil Plant}

\begin{abstract}
The production process of Biodiesel from castor oil plants is analyzed using the Supply Chain Operations Reference Model (SCOR). The different levels of the process, the key performance indicators, the attributes and the logistic operations carried out throughout the chain were analyzed. The usefulness of applying the SCOR model is determined by the identification of the components of the chain, highlighting strengths and weaknesses of external and internal relations of the logistics. From this perspective it was observed that the implementation of the SCOR model makes more efficient the logistics operations through the whole chain.
\end{abstract}

Keywords: SCOR, biodiesel, biofuels, castor oil plant, supply chains 


\section{INTRODUCCIÓN}

En un mundo con exigencias cada vez mayores desde el punto de vista energético y ambientalista, buscar soluciones para el problema de la energía, es un gran reto a emprender. Los servicios que la energía puede proveer, como iluminación, calefacción, cocción de alimentos, transporte motorizado, bombeo de agua, que entre otros, son esenciales para el desarrollo social y económico de la especie humana. Los servicios energéticos tienen un efecto multiplicador sobre los servicios de salud, educación, transporte, telecomunicaciones, agua y alcantarillado, así como sobre las inversiones y la productividad y rentabilidad de las actividades generadoras de ingresos, como la agricultura y la industria, sean éstas del sector de bienes y servicios (Korbitz, 1999). Por ello, la generación de energía a partir de los llamados procesos agroenergéticos, que producen Biocombustibles como el etanol y biodiesel, han contribuido a que los principales motivos que han llevado a los diferentes países a impulsar la producción de biodiesel han sido (Friedrich, 2004): i) una mayor seguridad en el abastecimiento energético; ii) la reducción de la dependencia de fuentes de energía de origen fósil; iii) la reducción de las emisiones de gases de efecto invernadero; iv) la reducción de emisiones dañinas de efecto local; la protección del suelo mediante el uso de productos biodegradables; v) la reducción de peligros a la salud mediante el uso de productos no tóxicos; y vi) la minimización de los excedentes de la producción agraria.

La Gestión de la cadena de suministro en la actualidad abarca infinidad de actividades características y únicas en la distribución que las empresas manejan, desde la recepción de materias primas hasta que se genera un producto terminado, permitiendo una mejor prestación de servicio al cliente y de la cadena de valor, a través de la gestión de los flujos de información, de productos y financiero. Dicha gestión, permite competir con éxito en los mercados actuales, gracias al resultado que produce la conjunción de los objetivos de la CS y la implantación de mejores prácticas en sus diferentes áreas. Actualmente, ésta es un elemento clave para la competitividad de las empresas debido a la importancia que tiene en los resultados empresariales, a través del margen de beneficio, calidad de productos y servicios, satisfacción del cliente y plazos de entrega. La CS engloba los procesos de negocio, las personas, la organización, la tecnología y la infraestructura física que permite la transformación de materias primas en productos y servicios intermedios o terminados que son ofrecidos y distribuidos al consumidor para satisfacer su demanda (Stadtler, 2005).

La posibilidad de descentralizar la producción de combustibles, el nuevo impulso a la agricultura, la creación de puestos de trabajo, y la soberanía energética, convierten al biodiesel; en un producto acorde a la solución de la escasez de combustibles y reducción de emisiones contaminantes (Friedrich, 2004); siendo de naturaleza renovable, y trayendo consigo la reducción de emisiones de efecto invernadero que puede tener con un manejo adecuado, y la significativa reducción de otro tipo de emisiones contaminantes durante su combustión, que con la utilización de modelos y metodologías validadas en aplicaciones a procesos del sector productivo, es lo que definió el marco de referencia para la aplicación de herramientas como SCOR, con el propósito de lograr identificar y definir la cadena de suministro en el campo de los biocombustibles. Entre los países más desarrollados y signatarios del Protocolo de Kyoto, la cuestión ambiental es el factor determinante para el empleo de los biocombustibles en general y del biodiesel como parte de ellos. Países que poseen gran capacidad de producción de oleaginosas también están efectuando esfuerzos para empezar la producción de biodiesel, la que ocupa mayor importancia entre los biocombustibles en la Unión Europea, con una participación en el mercado de alrededor del 80\% comparado al bioetanol con alrededor del 20\% (Von Lampe, 2006). La Unión Europea lidera el mundo en el desarrollo del biodiesel. Alemania, Francia e Italia son los mayores productores de este biocombustible y en la producción de bioetanol, sobresalen España, Francia, Polonia y Suecia. En América Latina, Brasil es el país que posee la mayor producción de biocombustibles con gran predominio del bioetanol de caña de azúcar. En México, la información más consistente sobre las perspectivas de uso del biodiesel se encuentra en un estudio de factibilidad conducido por la GTZ y el BID (2006). En resumen, el estudio alerta el hecho que México aún importa la mayoría de sus semillas oleaginosas, por lo que en caso de una mayor producción nacional de semillas deberán ser aprovechadas en la producción de aceites comestibles. 
Por otro lado, Colombia empezó un programa de uso de biodiesel y está cumpliendo las etapas previstas por la Unidad de Planeación Minero Energética del Ministerio de Minas y Energía. En septiembre de 2005 se completó la regulación de calidad para el producto (especificaciones técnicas). Las definiciones gubernamentales para señales de precios se concluyeron en diciembre de 2005. El desarrollo de pruebas estuvo previsto para el período julio de 2005 y julio de 2006, con caracterización de mezclas, pruebas en laboratorio y ruta. El reglamento de la logística estuvo previsto para junio de 2006. Los ajustes finales de la regulación se dieron para julio de 2007 y el inicio del programa en fase comercial en junio de 2008. Colombia usa el aceite de palma para producir el biodiesel y se están ampliando las plantaciones para ello; adicionalmente en el país se están haciendo investigaciones sobre el uso de Higuerilla y Jatropha Curcas (Ministerio de Minas y Energía, 2009).

Debido a la naturaleza compleja y dinámica de las organizaciones y procesos, los modelos son necesarios para entender el comportamiento de las mismas y diseñar los nuevos sistemas así como mejorar el funcionamiento de los existentes (Sanchis, 2009). SCOR es un modelo de referencia, es decir no tiene en cuenta modelos matemáticos, ni heurísticos, se basa en el manejo de indicadores, con el fin de analizar, comparar y obtener la mejor estrategia de mejoramiento, se trata de directrices o estándares de carácter multisectorial, dirigidas a avanzar en la gestión de estos procesos. El modelo SCOR fue creado en 1997 por el Consejo de Cadenas de Suministro de Estado Unidos, creando por primera vez un modelo de referencia de procesos, caracterizándose por ser un modelo relativamente nuevo y utilizado en pocas empresas, las cuales en su mayoría hacen parte del Consejo de Cadenas de Suministro o SCC. El objetivo principal del SCC es conseguir que las compañías involucradas en la Cadena de Suministro, mejoren el rendimiento de la misma. Una de las vías para conseguirlo es ayudando a las empresas y organizaciones a desarrollar sistemas basados en experiencias de éxito, lo que tendrá como resultado unas mejores relaciones con los clientes y proveedores, integrando de esta manera, toda la cadena de abastecimiento (SCOR, 2008).

Los objetivos de la presente investigación son: 1) Proponer un plan estratégico-logístico basado en el modelo SCOR, para fortalecer la cadena de suministro en los procesos de producción de biodiesel a partir de higuerilla, aplicado a procesos de obtención en Colombia; 2) Planificar eficientemente la capacidad instalada, fortaleciendo el proceso con el uso del modelo referencial SCOR del Supply Chain Council; y 3) Identificar oportunidades de mejora en el desempeño de la cadena de suministros tales como: reducción de costos, reducción de tiempos de ciclo, análisis de redes de distribución y transporte y mejora de niveles de servicio, a partir de la aplicación del Modelo SCOR (Figura 1). Para lograr el cumplimiento de estos objetivos, se busca el beneficio para toda la cadena y, a partir del conjunto, llegar a los beneficios individuales de cada uno de los eslabones, en contra del modelo tradicional, en el que cada componente buscaba los beneficios de forma individual. Si se cumplen estas premisas, será en principio factible la aplicación de los modelos de gestión del conocimiento a la cadena de suministro (Capo-Vicedo et al., 2007), tomando como metodología la aplicación del modelo SCOR.

\section{DISEÑO METODOLÓGICO}

El Supply Chain Operations Reference model (SCOR), como Supply Chain Management (SCM), provee una directriz o lineamiento para el mejoramiento de procesos y practicas relacionadas con la cadena de abastecimiento. El procedimiento para la aplicación del modelo SCOR consiste en analizar las bases de competencia, configurar la cadena de abastecimiento, alinear los niveles de desempeño, prácticas y sistemas e implementar los procesos y sistemas de la cadena de abastecimiento (SCOR, 2008). Los niveles del modelo SCOR con la definición de los procesos, son: Procesos de planeación: Desarrollo del Proyecto (Plan), Procesos Fuente: Materias primas o insumos para el proceso productivo (Source), Procesos de transformación: Obtención del biodiesel de Higuerilla (Make), Procesos de entrega: Distribución, transporte y comercialización (Delivery), y Procesos post-delivery: Para reprocesos, logística inversa, otros productos o subproductos (Return). 
Para este proyecto, la información de campo se levantó en el centro de investigación de Corpoica en Colombia, el centro está destinado a la investigación de Biodiesel de Higuerilla (Ricinus Communis). Esto hizo con el fin de generar información fiable y útil para el análisis de los procesos en el manejo actual de la cadena de suministro y la identificación de las actividades para su medición a fin de generar un mejoramiento continuo. Con la información obtenida, se caracterizó la cadena de suministro con la aplicación del modelo SCOR (Supply Chain Operations Reference Model), ésta abarca procesos de negocio, indicadores de gestión o KPI (Key Performance Indicators), mejores prácticas, oportunidades de mejora, medición del desempeño de la cadena y el uso adecuado de tecnologías para apoyar la comunicación entre los socios que hacen parte de la cadena de suministro (Bolstorff y Rosembaum, 2007).

Para la metodología de aplicación, se tomó en cuenta el ambiente del sector productivo del Biodiesel, en donde se consideraron los siguientes aspectos para el desarrollo del modelo propuesto (Chen y Wang, 1997), de acuerdo con la información recopilada del proceso, en el cual se utiliza como materia prima la Higuerilla para producir biodiesel (Figura 1): i) Capacidad de cultivo o de producción con las variables que actúan en este subsistema; ii) Comportamiento en el mercado de la materia prima; iii) Redes de distribución y transporte existentes en el sector; iv) Procesamiento para la producción del aceite de ricino, que es la base para el Biodiesel; ) Proceso de producción del Biodiesel y manejo de los subproductos en la industria; y vi) Comercialización y distribución del Biocombustible, Biodiesel y su utilización en el mercado del sector de transportes.

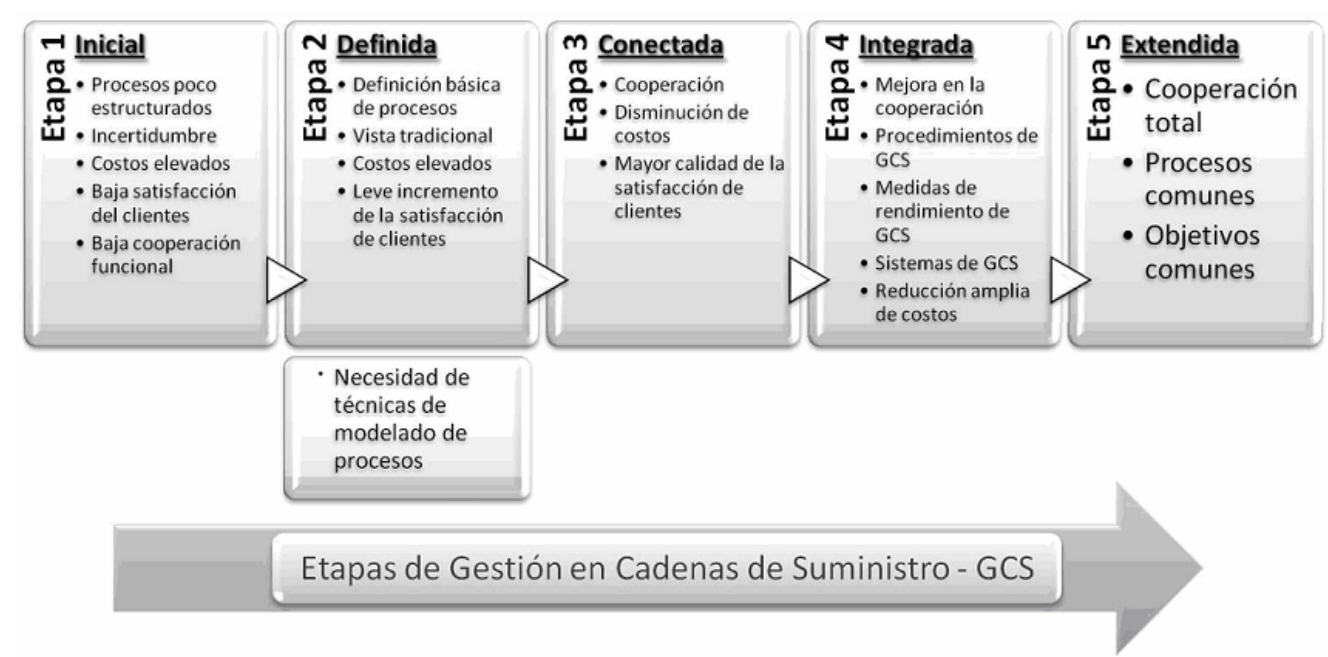

Fig. 1: Etapas de Gestión de la Cadena de Suministro (Harmon, 2003).

El modelo SCOR, que abarca desde las relaciones con los clientes (entrada de órdenes hasta el pago de las facturas), las transacciones físicas de materiales (desde los proveedores de los proveedores (Suppliers) hasta los clientes de los clientes (Customers), incluyendo equipos, suministros, repuestos, productos a granel y software.) y todas las relaciones con el mercado (desde la demanda agregada hasta el cumplimiento de cada Orden) (Calderón y Lario, 2005). El modelo se basa en indicadores de gestión o KPI'S, los cuales tienen también 5 atributos principales de representación de objetivos de rendimiento competitivo, que hacen parte de los niveles de especificación que presenta el modelo (Ballou, 2004):

Reliability (Confiabilidad de la entrega): Con el uso de este atributo, se busca enviar el producto esperado (biodiesel) en el lugar y momento adecuado (puntos de entrega o distribución), teniendo en cuenta las condiciones que se esperan (rendimiento como combustible), y en el empaque y la cantidad adecuada, con la documentación requerida y al cliente indicado.

Agility (Agilidad): Se busca mantener una ventaja competitiva (frente a otro biodiesel obtenido de otros productos como el aceite de palma y otras oleaginosas o como la jatropha curcas), mediante la agilidad o rapidez de respuesta que tenga la cadena de suministro para responder ante cambios que se den en el mercado (incrementos en la demanda o cambios en la producción debidos a cambios de las condiciones del clima que alteran los ciclos de cultivos). 
Responsiveness (Capacidad de respuesta): Hace referencia a la velocidad que tiene la cadena de suministro, para proporcionar productos a los clientes (por demanda cambiante).

Cost (Costos): Hace referencia a los costos que se generan en las operaciones que se dan dentro de la cadena de suministro (por insumos como la ricinus cummnis o higuerilla).

Assets (Administración de activos): Garantiza la efectividad organizacional, en el manejo de todos los activos, con el fin de lograr satisfacer la demanda (buscando fortalecer los procesos productivos a partir de la mejora en la obtención de insumos por rendimiento y en mejora de equipos para el proceso).

Los tres primeros atributos se caracterizan por ser puntos de vista del entorno exterior o customer facing, mientras que los dos últimos, costos y administración de activos son puntos de vista internos o internal facing (Calderón y Lario, 2005). Para el Supply Chain Council, los niveles de detalle o caracterización que presenta SCOR (2008), en los cuales presenta los atributos previamente mencionados son: Nivel Superior en el cual se especifican los Tipos de Procesos que se utilizan dentro de la empresa, en los cuales se indican sus bases de competencia y objetivos de rendimiento competitivo teniendo en cuenta los 5 atributos mencionados; seguidamente se encuentra el Nivel de Configuración en la cual se trabajan las categorías de Procesos, en el segundo nivel se consideran 26 Categorías de Procesos que Corresponden: 5 a Plan, 3 a Aprovisionamiento, 3 a Manufactura, 4 a Distribución, 6 a Devolución (3 de Aprovisionamiento y 3 de Distribución), y 5 a Apoyo. Las 5 primeras son tipo Planificación, las 16 intermedias son tipo Ejecución y las 5 últimas son tipo Apoyo (Calderón y Lario, 2005).

\section{MODELO METODOLÓGICO}

Para aplicar el modelo se identificaron los diferentes niveles en los que está estructurado el modelo, de acuerdo al SCC. De estos niveles, del uno al tres, se tomaron las operaciones, transacciones, indicadores y atributos que aplican a la cadena de suministro, propia de proceso (Figura 2). Se identificaron los componentes de la Cadena de Suministro (Figura 3) del proceso de producción de biodiesel de higuerilla, la que se encuentra estructurada por los siguientes eslabones a partir de subprocesos:

Eslabón primero del subproceso de cultivo de la higuerilla (Source), desde la siembra hasta la recolección del fruto, como insumo para el siguiente eslabón; el segundo eslabón lo componen las operaciones logísticas del subproceso de obtención del aceite de ricino (Make), que es el componente principal en la obtención del biodiesel. En este subproceso, se tienen en cuenta operaciones de los llamados co-productos, ya que representan un área de oportunidad desde el punto de vista de aplicación de operaciones en logística inversa o reversa, a partir de la detoxificación de estos, definiendo varias clases de co-productos; y el tercer eslabón (Deliver), que es el almacenamiento, despacho, transporte, distribución y entrega del biodiesel (biocombustible) al consumidor final en las estaciones de tanqueo. El componente de SCOR Model, Return o devoluciones, se ubica en los subprocesos de Source para cultivo de la higuerilla y en el Make o producción del biodiesel, ya que es aquí en estos dos subprocesos, donde se generan las operaciones de devoluciones.

El quinto elemento del SCOR Model, Plan, se encuentra presente en toda la cadena de suministro (Figura 5), desde la planeación y control de los cultivos, hasta la entrega al cliente o consumidor final, proceso que se retroalimenta de manera constante por medio de la evaluación de los indicadores de desempeño logístico (Tabla 1). De los indicadores de desempeño que sugiere y define el SCOR Model, en el proceso se listan los que afectan de manera directa a la cadena de suministro, debido a la relación entre las diferentes operaciones logísticas y el impacto que se puede tener sobre los atributos, en cuanto a fiabilidad, responsabilidad, flexibilidad, costos y activos (Tabla 1).

El Mapa de Procesos de SCOR y tipos de categorías, se definió de acuerdo a las operaciones que aplican del SCOR Model y sus actividades (Tabla 2), para el nivel dos (de configuración), donde 
se identifican los aspectos que intervienen en la cadena de suministro del biodiesel. Se identificaron los componentes de la Cadena de Suministro del proceso de producción de biodiesel de higuerilla, la que se encuentra estructurada por los siguientes eslabones a partir de subprocesos (Figura 4). El Mapa de Procesos de SCOR y tipos de categorías, se definió de acuerdo a las operaciones que aplican del modelo y sus actividades (Tabla 3), para el nivel dos (de configuración), donde se identifican los aspectos que intervienen en la cadena de suministro del biodiesel:

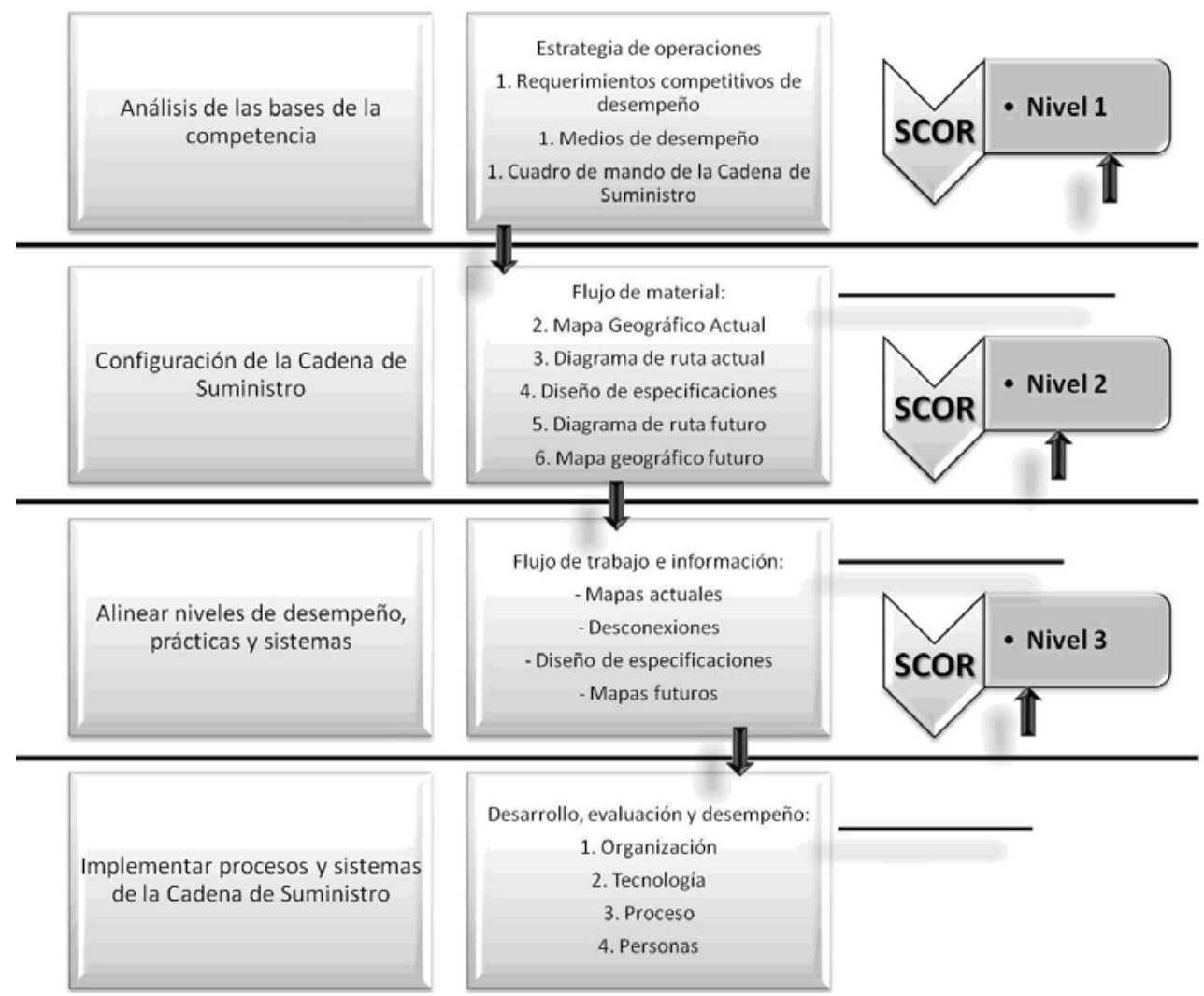

Fig. 2: Plan de proyecto (SCOR, 2008).

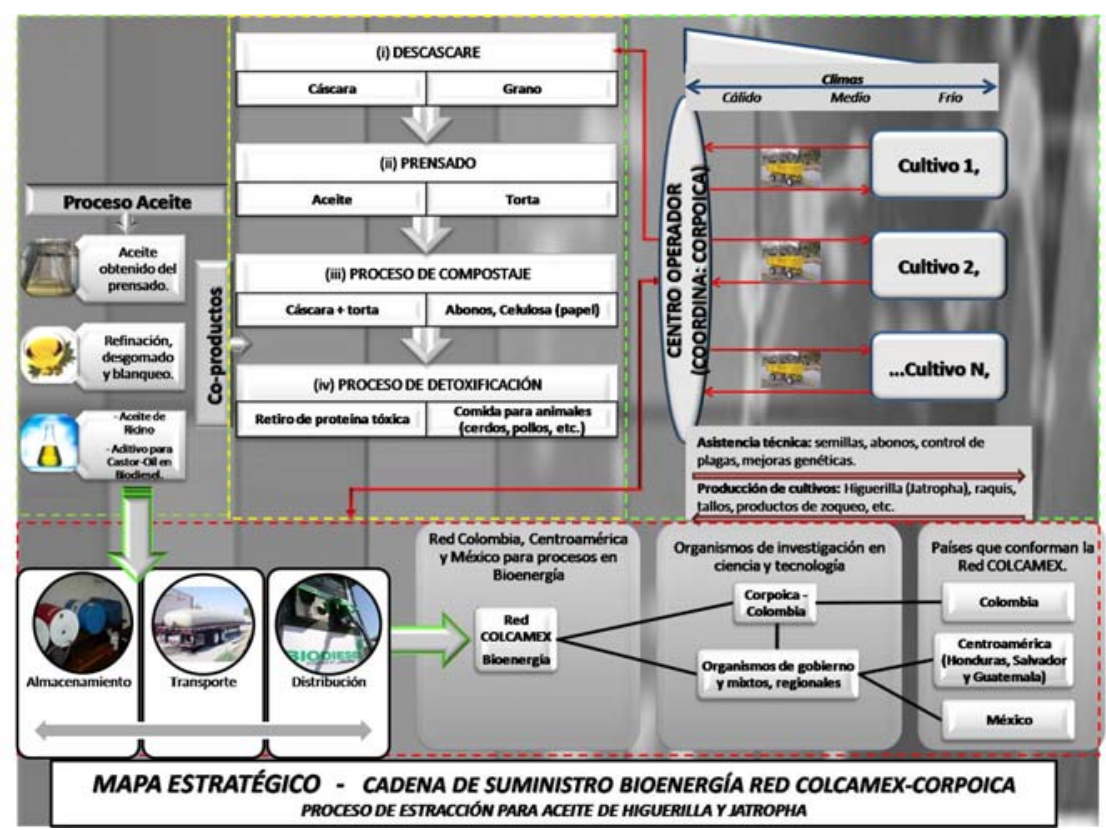

Fig. 3: Cadena de Suministro de Biodiesel de Higuerilla (Elaboración propia, 2010). 
Tabla 1: Indicadores de Primer Nivel (Adaptado de SCOR, 2011)

\begin{tabular}{|c|c|c|c|c|c|}
\cline { 2 - 6 } \multicolumn{1}{c|}{} & \multicolumn{2}{c|}{ De cara al Mercado (Cliente) } & \multicolumn{2}{c|}{ De cara al Interior } \\
\hline Atributos de Desempeño & Fiabilidad & Responsabilidad & Flexibilidad & Costos & Activos \\
\hline Desempeño en la entrega & $\mathrm{X}$ & & & & \\
\hline Tasa de surtido (Fill Rate) & $\mathrm{X}$ & & & & \\
\hline $\begin{array}{c}\text { Orden perfectamente cumplida } \\
\text { (perfect Order Fulfillment) }\end{array}$ & & & & & \\
\hline $\begin{array}{c}\text { Tiempo de Ejecución para } \\
\text { Cumplimiento Perfecto de la Orden } \\
\text { (Order Fulfillment Lead Time) }\end{array}$ & & $\mathrm{X}$ & & & \\
\hline $\begin{array}{c}\text { Tiempo de Respuesta de la Cadena } \\
\text { de Suministro }\end{array}$ & & & $\mathrm{X}$ & & \\
\hline Flexibilidad de la Producción & & & $\mathrm{X}$ & & \\
\hline $\begin{array}{c}\text { Costos Administrativos de la } \\
\text { Cadena de Suministro }\end{array}$ & & & & $\mathrm{X}$ & \\
\hline Costo de Ventas & & & & $\mathrm{X}$ & \\
\hline Valor Agregado de la Productividad & & & & $\mathrm{X}$ & \\
\hline Costo de Garantía y Retornos & & & & & $\mathrm{X}$ \\
\hline Tiempo del Ciclo de Pagos & & & & & $\mathrm{X}$ \\
\hline Días de la Oferta de Inventarios & & & & & \\
\hline Cambio de Activos & & & & & \\
\hline
\end{tabular}

Tabla 2: Procesos y sus categorías para el Nivel dos (Adaptado de SCOR, 2011).

\begin{tabular}{|c|c|c|c|c|c|c|c|}
\hline & \multirow{2}{*}{\multicolumn{5}{|c|}{ Procesos SCOR Model }} & \\
\hline & & & & & & & \\
\hline & & PLAN & SOURCE & MAKE & DELIVER & RETURN & \\
\hline \multirow{3}{*}{ 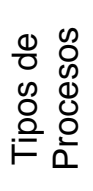 } & Planning & P1 & P2 & P3 & P4 & P5 & \multirow{3}{*}{ 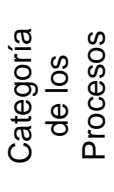 } \\
\hline & Execution & & S1, S2 & M1, M2 & D1, D2 & SR1, SR2, DR1, DR2 & \\
\hline & Enable & EP1-EP10 & ES1-ES10 & EM1-EM9 & ED1-ED9 & ER1-ER9 & \\
\hline
\end{tabular}

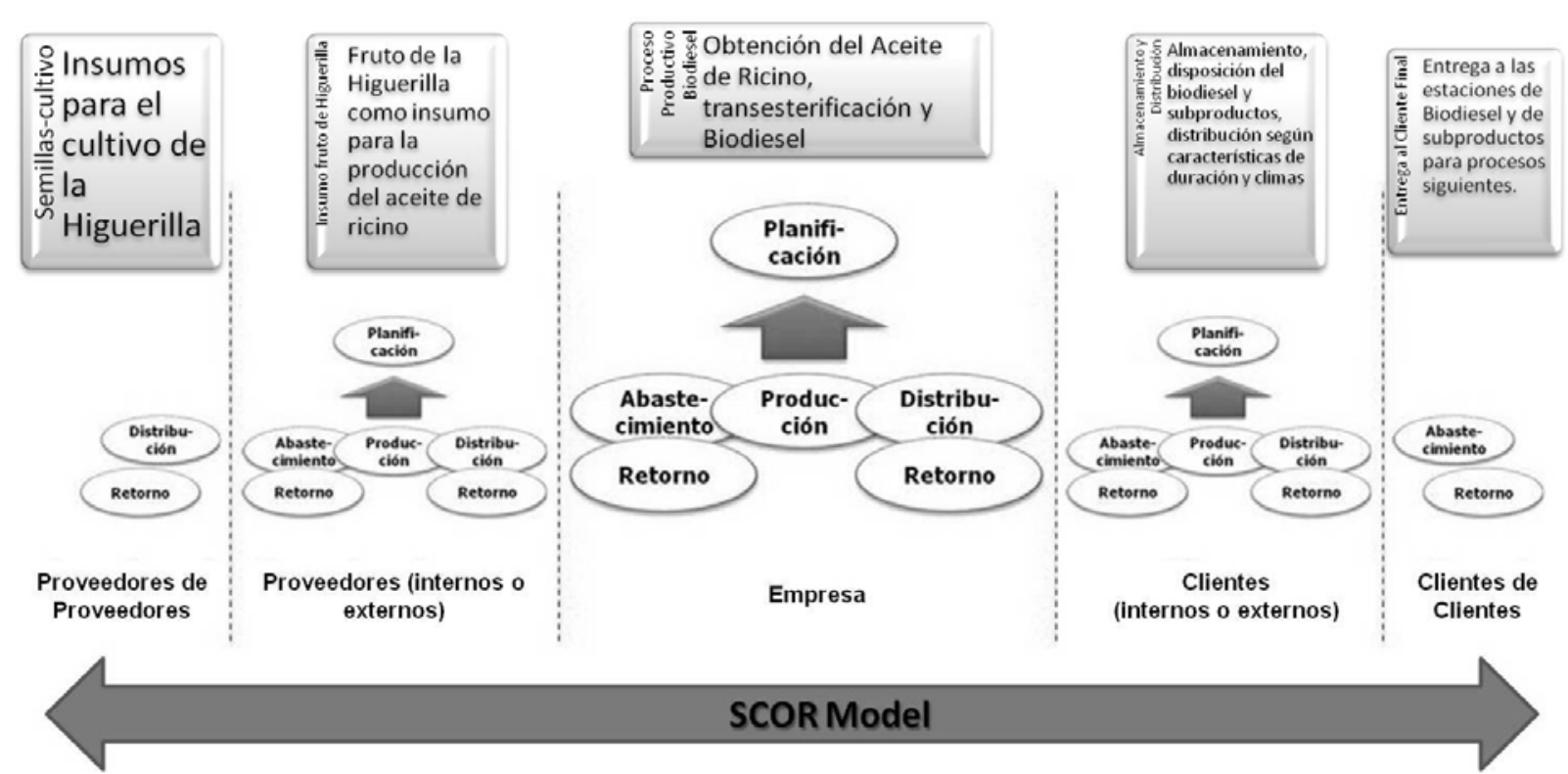

Fig. 4: Cadena de Abastecimiento en la Producción de Biodiesel de Higuerilla (Adaptado de SCOR, 2011). 
Tabla 3: SCORCard del proceso de producción del Biodiesel de Higuerilla por Atributos (Adaptado de SCOR, 2011).

\begin{tabular}{|c|c|c|c|c|c|}
\hline \multicolumn{6}{|c|}{ SCORCard Biodiesel de Higuerilla } \\
\hline $\begin{array}{l}\text { Atributo o } \\
\text { Categoría }\end{array}$ & Métrica Nivel 1 & Actual & Meta & Brecha & Oportunidad \\
\hline \multirow{4}{*}{$\begin{array}{l}\text { Confiabilidad } \\
\text { Cadena de } \\
\text { Suministro } \\
\text { (Despacho) }\end{array}$} & $\begin{array}{l}\text { Cumplimiento perfecto de } \\
\text { órdenes }\end{array}$ & $78 \%$ & $97 \%$ & $19 \%$ & \multirow{4}{*}{$50 \%$} \\
\hline & $\begin{array}{l}\% \text { de órdenes entregadas } \\
\text { completas }\end{array}$ & $80 \%$ & $100 \%$ & $20 \%$ & \\
\hline & Documentación & $100 \%$ & $100 \%$ & $0 \%$ & \\
\hline & Condición perfecta & $97 \%$ & $97 \%$ & $0 \%$ & \\
\hline \multirow{4}{*}{$\begin{array}{l}\text { Fexibilidad } \\
\text { Cadena de } \\
\text { Suministro }\end{array}$} & $\begin{array}{l}\text { Tiempo promedio del proceso de } \\
\text { compra de insumos (días) }\end{array}$ & 3 & 3 & 0 & \multirow{3}{*}{$\begin{array}{c}\text { Doblar la } \\
\text { producción de } \\
\text { Biodiesel }\end{array}$} \\
\hline & $\begin{array}{l}\text { Tiempo promedio del proceso de } \\
\text { producción de biodiesel/litro } \\
\text { (días) }\end{array}$ & 9.0 & 4.5 & -4.5 & \\
\hline & $\begin{array}{l}\text { Tiempo promedio de entrega } \\
\text { (horas) }\end{array}$ & 12 & 12 & 0 & \\
\hline & $\begin{array}{l}\text { Tiempo de rotación en } \\
\text { estaciones (días/lt) }\end{array}$ & 0.6734 & 1 & 0.3266 & $\begin{array}{l}\text { Menor cantidad } \\
\text { de despachos }\end{array}$ \\
\hline \multirow{8}{*}{$\begin{array}{l}\text { Agilidad } \\
\text { Cadena de } \\
\text { Suministro }\end{array}$} & $\begin{array}{l}\text { Días para incrementar la } \\
\text { producción de todo el proceso de } \\
\text { biodiesel en un } 20 \%\end{array}$ & 1 & 0.808 & -0.1919 & \multirow{8}{*}{ Varía } \\
\hline & $\begin{array}{l}\text { Máximo \% de incremento en la } \\
\text { cadena }\end{array}$ & $0 \%$ & $15 \%$ & $15 \%$ & \\
\hline & $\begin{array}{l}\text { Días para incrementar el } \\
\text { aprovisionamiento en un } 20 \%\end{array}$ & 3 & 3 & 0 & \\
\hline & $\begin{array}{l}\text { Días para incrementar la } \\
\text { producción de biodiesel en un } \\
20 \%\end{array}$ & 1 & 0.808 & -0.1919 & \\
\hline & $\begin{array}{l}\text { Días para incrementar la entrega } \\
\text { o distribución en un } 20 \%\end{array}$ & 1 & 1 & 0 & \\
\hline & $\begin{array}{l}\text { Máximo \% de incremento en la } \\
\text { proveeduría }\end{array}$ & $25 \%$ & $25 \%$ & $0 \%$ & \\
\hline & $\begin{array}{l}\text { Máximo \% de incremento en la } \\
\text { producción }\end{array}$ & $0 \%$ & $15 \%$ & $15 \%$ & \\
\hline & $\begin{array}{l}\text { Máximo \% de incremento en la } \\
\text { distribución y entrega }\end{array}$ & $20 \%$ & $20 \%$ & $0 \%$ & \\
\hline \multirow{4}{*}{$\begin{array}{l}\text { Costos de la } \\
\text { Cadena de } \\
\text { Suministro }\end{array}$} & $\begin{array}{l}\text { Costo de mantener la cadena de } \\
\text { suministro }\end{array}$ & $100 \%$ & $90 \%$ & $-10 \%$ & \multirow{4}{*}{$10 \%$} \\
\hline & Costo de planear & $80 \%$ & $50 \%$ & $-30 \%$ & \\
\hline & Costo de producir & $10 \%$ & $10 \%$ & $0 \%$ & \\
\hline & Costo de entregar o distribuir & $10 \%$ & $8 \%$ & $-2 \%$ & \\
\hline \multirow{2}{*}{$\begin{array}{l}\text { Manejo de } \\
\text { Activos en la } \\
\text { Cadena de } \\
\text { Suministro }\end{array}$} & $\begin{array}{l}\text { Ciclo de flujo de efectivo para } \\
\text { Biodiesel (días) }\end{array}$ & 11.5 & 9 & -2.5 & \multirow{2}{*}{ Mayor liquidez } \\
\hline & $\begin{array}{l}\text { Ciclo de flujo de efectivo para } \\
\text { Proveedores (días) }\end{array}$ & 30 & 30 & 0 & \\
\hline
\end{tabular}




\section{RESULTADOS}

Se encontraron las siguientes ventajas en la aplicación del SCOR Model a la cadena de abastecimiento de biodiesel de higuerilla: Es un modelo que presenta una metodología formal para su identificación, diseño, análisis y evaluación de las operaciones de la cadena de suministro del biodiesel de higuerilla. En la investigación que se hizo de búsqueda de referencias y antecedentes sobre el tema de aplicación de SCOR a cadenas de suministro de biodiesel, no se encontró evidencia en las fuentes consultadas, sobre investigación aplicada en esta metodología o similar, que incluya el análisis y operación de la cadena de suministros desde el proveedor del proveedor, hasta el cliente del cliente para cadenas de biodiesel. Esta búsqueda se realizó en fuentes provenientes de bases de datos como EBSCO, Redalyc, Scielo, Información Tecnológica y Actualidad y Nuevas Tendencias, entre otras. El uso de la metodología SCOR educa a la organización, pero más que eso a los actores del proceso de producción de biodiesel de higuerilla, acerca de la importancia de fortalecer y definir la cadena de suministro.

La práctica de la evaluación del rendimiento en las cadenas de suministro es muy escasa, por lo que diversos factores contribuyen a tal hecho. La cultura tradicional de medición en los procesos de Biocombustibles, conduce a tomar medidas aisladas en puntos específicos de la cadena, ubicándola lejos de imprimirle un carácter sistémico (Campos et al., 2004). Se debe guiar a los procesos de la cadena de suministro al análisis competitivo para la definición de oportunidades de negocio, haciéndolo más competitivo y fortaleciendo las relaciones entre la logística interna y externa. Una desventaja del modelo SCOR que fue encontrada, se puede describir como: Es difícil encontrar información acerca del modelo, se percibe una carencia de casos prácticos en Latinoamérica que ejemplifiquen la implementación del modelo SCOR, con mayor énfasis, en el campo de los biocombustibles o cadenas de suministro de energías alternativas.

\section{CONCLUSIONES}

La aplicación del Modelo SCOR, generó como resultado el bajo nivel de identificación de las operaciones logísticas de toda la cadena de suministro para los procesos de producción de biodiesel de higuerilla. No existe una relación basada en confianza entre las partes de la logística externa e interna, que se basen en confianza, pues se basa más en las leyes de oferta-demanda y comportamientos de acaparamiento de insumos o materias primas por parte de los cultivadores de estos, debido a la inexistencia de relaciones ganar-ganar. La presente investigación, con los resultados de este análisis de la cadena de suministro, basado en el SCOR Model, deja abiertas posibilidades para futuras investigaciones, basadas en el modelamiento o simulación de la cadena de suministro que podrían ser herramientas que se sustentan en dinámica de sistemas.

\section{AGRADECIMIENTOS}

Este proyecto ha sido realizado con el apoyo académico y financiero de CONACyT y la UPAEP (México), el apoyo técnico y académico de EAFIT y Corpoica (Colombia), como estancia doctoral.

\section{REFERENCIAS}

Ballou, R. H. LOGÍSTICA, Administración de la Cadena de Suministros. Pearson, (2004).

Banco Interamericano de Desarrollo (BID), ME-T1007 - ATN/DO-9375-ME, y Deutsche Gesellschaft für Technische Zusammenarbeit (GTZ) GmbH - Cooperación técnica alemana, Potenciales y Viabilidad del Uso de Bioetanol y Biodiesel para el Transporte en México, (2006).

Bolstorff, P. y Rosembaum, R. Supply Chain Excelence, Nueva York, Amacom, (2007).

Calderón J. y Lario, E. Análisis del Modelo SCOR para la Gestión de la Cadena de Suministro, $9^{\circ}$ Congreso de Ingeniería de Organización, Gijón (2005).

Campos, J.; Taboada, C. y Chalmeta, R. Metodología para la Evaluación del Rendimiento de la Cadena Logística. Inf. tecnol., .15(4) 77-84 (2004) 
Capo-Vicedo, J.; Tomas-Miquel, J.; Exposito-Langa, M. La Gestión del Conocimiento en la Cadena de Suministro: Análisis de la Influencia del Contexto Organizativo.Inf. tecnol.. 18(1) pag? (2007).

Chen M. y Wang, W. A linear programming model for integrated steel production and distribution planning, International Journal of Operations \& Production Management, Vol. 17 No. 6, pp. 592610 (1997).

Friedrich S. A World wide review of the commercial production of biodiesel - A technological, economic and ecological investigation based on case studies. Schriftenreihe Umweltschutz und Ressourcenökonomie, Band 41. Viena: Institut für Technologie und nachhaltiges Produktmanagement der Wirtschaftsuniversität, p. 150 (2004).

Harmon, P.; Business Process Change: A Manager's Guide to Improving, Redesigning, and Automating Processes. Morgan Kaufmann, San Francisco, USA (2003).

Korbitz W. Biodiesel production in Europe and North America, an encouraging prospect, Renewable Energy, 16, 1-4:1078-1083 (1999).

Ministerio de Minas y Energía, 2009. Colombia. Disponible en: http://www.minminas.gov.co/ minminas/index.jsp?cargaHome=2\&opcionCalendar=733\&tipo_comunicado=COMUNICADOS\%20 SECTOR [Consultado: 10 de noviembre] (2009).

Sanchis, R.; Poler, R. y Ortiz, Ángel. Técnicas para el Modelado de Procesos de Negocio en Cadenas de Suministro. Inf. tecnol., 20(2) pag (2009)

Stadtler, H. Supply Chain Management and Advanced Planning - Basics, Overview and Challenges. European Journal of Operational Research, 163(3), pp. 575-588. (2005).

Supply Chain Operations Reference Model, SCOR. Supply Chain Council. Version 9, 2008.

Von Lampe, M. Agricultural market impacts of future growth in the production of biofuels, OCDE Report AGR/CA/APM (2005) 24/FINAL, 01/02 (2006). 